\title{
Collapsing singletons may boost signal for associating rare variants in sequencing study
}

\author{
Wei Wang, Zhi Wei \\ From Genetic Analysis Workshop 18 \\ Stevenson, WA, USA. 13-17 October 2012
}

\begin{abstract}
Advances in next-generation sequencing technology have made it possible to comprehensively interrogate the entire spectrum of genomic variations including rare variants. They may help capture the remaining genetic heritability which has not been fully explained by previous genome-wide association studies. Here we performed a gene-based genome-wide scan to identify hypertension susceptibility loci in analysis of a whole genome sequencing cohort of 103 unrelated individuals. We found that collapsing singletons may boost signals for associating rare variants and identified SETX statistically significant by a genome-wide gene-based threshold $\left(p\right.$ value $\left.<5.0 \times 10^{-6}\right)$. The function of SETX in hypertension may be worthy of further investigation.
\end{abstract}

\section{Background}

Assuming the "common disease-common variant" hypothesis, genome-wide association studies (GWAS) have successfully identified hundreds of common variants that contribute to human traits and diseases [1]. These common variants, however, account for only a small fraction of disease or trait heritability. On the other hand, rare variants, having minor allele frequencies (MAFs) between $0.1 \%$ and $1 \%$, may be functionally relevant and causal for a larger proportion of inheritable variability $[2,3]$.

Recent advances in next-generation sequencing (NGS) technology have made it technically and economically feasible to capture the full spectrum of genomic variation. NGS provides a powerful tool for systematic exploration of common and rare variants in the entire genome, even in large population-scale studies [4]. However, pinpointing causal variants remains a major challenge, particularly for associating rare variants with complex traits [5]. There is a substantial need for computational methods that allow for efficient association analysis of rare variants. Several powerful approaches tailored for rare-variant association studies have been proposed [6-9]. These tests offer us a powerful tool to investigate rare variants in the entire genome.

\footnotetext{
* Correspondence: zhiwei@njit.edu

Department of Computer Science, New Jersey Institute of Technology, University Heights Newark, New Jersey 07102, USA
}

\section{Methods \\ Data set}

The Genetic Analysis Workshop 18 (GAW18) provided a whole genome sequencing data set for a hypertension cohort of 483 individuals. These samples were sequenced by Complete Genomics with approximately $60 \times$ coverage, and odd-numbered autosomes data were made available for analysis. After quality control, 464 individuals and 24 million single-nucleotide polymorphisms (SNPs) remained. Of those SNPs, more than 51\% had MAFs $<1 \%$, which was the focus of our analysis in this article. The longitudinal hypertension phenotypes were provided for up to 4 time points. Because our analysis was focused on binary traits, we treated individuals diagnosed with hypertension in any of the 4 times as cases. We extracted 103 genetically unrelated individuals with both phenotype data and sequencing data. We found 39 unaffected controls and 64 cases affected by hypertension.

\section{Preprocessing}

The variants were stored in VCF files. We preprocessed them as follows. To end up with rare variants, we filtered out SNPs that were present in dbSNP132 or MAFs $>1 \%$. We also filtered out SNPs with a genotype missing rate $>5 \%$. The remaining missing genotypes were resampled from nonmissing individuals. 


$$
X_{i}=\left\{\begin{array}{c}
1 \text { if any } x_{i, j}=1 \\
0 \text { otherwise }
\end{array}\right.
$$

Next, we grouped variants into sets based on RefSeq gene annotations [10], requiring SNPs lie between the RefSeq transcript start site and transcript end site. SNPs outside gene boundaries were not analyzed. In total, 10,148 genes from odd-numbered chromosomes were used. Finally, we collapsed singletons within each gene. A singleton was a variant being observed only once among all the samples. The rationale of collapsing singletons was that the distribution of singletons as 1 variable may reflect the association between genotype and phenotype. Hence, we created 1 supervariant for each gene by combining all the singletons within it using the following rules: for each sample, (a) the genotype was set to be 1 , if there was at least 1 variant observed; (b) otherwise, the genotype was set to be 0 .

\section{Rare-variant association tests}

We employed 3 recently published rare association tests, qMSAT [7], C-alpha [8], and CMC [9]. The qMSAT is a quality-weighted multivariate score association test that can utilize genotype quality information. However, genotype quality score information was not available in the GAW18 raw VCF files. Without utilizing quality information, the qMSAT test was equivalent to the linear sequence kernel association test (SKAT) [6], Sum of Squared U statistic test (SSU) [11], and C-alpha. The C-alpha test compared the assumed binomial distribution of rare variants in cases versus controls via a homogeneity test. CMC, a combined multivariate and collapsing method, collapsed variants in subgroups according to allele frequencies and combines these subgroups using Hotelling's $T^{2}$ test. For these 3 tests, we used permutation to evaluate association significance. Because permutation was computationally expensive, we utilized a 2-step strategy in searching and testing candidate loci. Specifically, we first used 1000 permutations, from which we can identify candidates with estimated $p$ value $<0.001$. Then for these candidates we conducted $10^{6}$ permutations so as to know if any loci were significant at a genome-wide gene-based threshold $(0.05 / 10,000=5.0 \times$ $10^{-6}$ ) using a Bonferroni assumption.

\section{Results}

After the preprocessing step, we obtained approximately 2.2 million rare variants, which were assigned to 10,148 genes for testing. We then performed the 3 tests, qMSAT, C-alpha, and CMC, using R (http:// www.r-project.org). The qMSAT, C-alpha, and CMC identified 10, 6, and 7 genes with an estimated $p$ value $<0.001$, respectively (Table 1 ). Only SETX revealed significance for all of the 3 methods. Using $10^{6}$ permutations, qMSAT, C-alpha, and CMC yielded more
Table 1 Genes with $p<0.001$ from at least 1 method using $10^{3}$ permutations

\begin{tabular}{|c|c|c|c|c|c|}
\hline Chr & Gene & $\begin{array}{c}\text { \# Variants } \\
\text { (singletons) }\end{array}$ & qMSAT & $\begin{array}{c}\text { C- } \\
\text { alpha }\end{array}$ & CMC \\
\hline chr1 & NUP210L & $674(221)$ & Y & & \\
\hline chr1 & USP1 & 51(19) & Y & Y & \\
\hline chr7 & CUL1 & $348(114)$ & Y & & \\
\hline chr9 & RAB14 & $88(32)$ & Y & & \\
\hline chr9 & SETX & $380(135)$ & Y & Y & Y \\
\hline chr11 & FLJ39051 & $44(11)$ & Y & & \\
\hline chr11 & GDPD5 & $338(104)$ & Y & & \\
\hline chr19 & GRIN3B & $24(8)$ & Y & & \\
\hline chr19 & LOC100505495 & 249(78) & Y & Y & \\
\hline chr19 & PSG5 & $111(26)$ & Y & & Y \\
\hline chr 5 & CXXC5 & $96(35)$ & & Y & \\
\hline chr15 & RCCD1 & $32(13)$ & & Y & \\
\hline chr17 & WSCD1 & $183(70)$ & & Y & \\
\hline chr17 & MLLT6 & 92(33) & & & Y \\
\hline chr1 & ATF6 & $576(173)$ & & & Y \\
\hline chr7 & ZNF775 & $69(21)$ & & & Y \\
\hline chr19 & LOC100128252 & $45(13)$ & & & Y \\
\hline chr5 & LOC728342 & 495(146) & & & Y \\
\hline
\end{tabular}

precise $p$ values of $2.0 \times 10^{-6}, 1.0 \times 10^{-6}$, and $6.0 \times 10^{-6}$, respectively, for SETX (Table 2). The CMC $p$ value was slightly higher than the genome-wide gene-based threshold, which was possibly a result of its lower power compared to qMSAT and C-alpha [7].

SETX locates in chr9:135,136,827-135,230,372 and is a relatively large gene among all the human genes. The length of SETX (93,545 base pairs [bp]) is far greater than the median number $(17,970 \mathrm{bp})$ of all the genes $(p$ value $<2.2 \times 10^{-16}$, one-sided Wilcoxon signed rank test). Although it contains 26 exons, the total length of coding regions is only 8,034 , suggesting that SETX includes large intronic regions. To pinpoint causal regions, we divided the 380 variants of SETX into 3 groups based on its functional annotations. Specifically, we applied ANNOVAR [12] to annotate the variants of SETX and grouped them into coding sequence regions (CDSs), untranslated regions (UTRs), and intronic regions (INTRONs) (see Table 2). We observed that a majority of rare variants were, indeed, from the intronic region. We tested these 3 regions using the same tests with $10^{6}$ permutations. We found that the UTR group and the CDS group were far from being significant, suggesting that they may be irrelevant. Another possible reason may be that there are very few variants in these categories. Because the INTRON group became more significant than the whole gene-based tests after excluding the variants from these 2 groups, we may conclude that causal variants locate in the intronic region of SETX. 
Table 2 Functional annotation and test of the rare variants in SETX

\begin{tabular}{cccccccc}
\hline \hline & & \multicolumn{2}{c}{$\boldsymbol{p}$ Value | OR | 95\% CI } & \multicolumn{2}{c}{$\boldsymbol{p \text { Value* }}$} \\
\hline Regions & \# Variants (singletons) & \multicolumn{2}{c}{ Fisher's exact test on supervariant ${ }^{\dagger}$} & \multicolumn{2}{c}{ qMSAT } & C-alpha & CMC \\
\hline SETX (CDS + UTR + INTRON) & $380(135)$ & $3.7 \times 10^{-6}$ & 8.8 & {$[3.12,27.43]$} & $2.0 \times 10^{-6}$ & $1.0 \times 10^{-6}$ & $6.0 \times 10^{-6}$ \\
CDS & $14(8)$ & 1.000 & 1.0 & {$[0.18,6.94]$} & 1.000 & 0.544 & 0.837 \\
UTR & $6(4)$ & 0.632 & 0.6 & {$[0.04,8.60]$} & 1.000 & 0.662 & 0.990 \\
INTRON & $360(123)$ & $8.8 \times 10^{-7}$ & 9.5 & {$[3.43,28.70]$} & $<1.0 \times 10^{-6}$ & $<1.0 \times 10^{-6}$ & $<1.0 \times 10^{-6}$ \\
\hline
\end{tabular}

${ }^{*} p$ Values were calculated using $10^{6}$ permutations.

${ }^{\dagger}$ Supervariant was defined by collapsing all the singletons.

We then sought to elucidate why and where the signal came from. To this end, several in-depth analyses for SETX were performed. First, the Fisher's Exact test was conducted on the super feature we created by collapsing singletons. We found that, by collapsing all the 135 singletons on SETX, it achieved a very significant $p$ value $\left(3.7 \times 10^{-6}\right)$, together with $\mathrm{OR}=8.8$ and $95 \% \mathrm{CI}=[3.12$, 27.43] (see Table 2). This explained why SETX could be detectable under such a small sample size. We obtained more significance when testing the super feature with only singletons within the intronic regions ( $p$ value $=$ $8.8 \times 10^{-7}, \mathrm{OR}=9.5$, and $\left.95 \% \mathrm{CI}=[3.43,28.70]\right)$, which was consistent with the results from 3 rare variant association tests. Second, we checked each rare variant and singleton individually by performing the same test. It turned out that none of them were significant, when the minimum $p$ value was merely 0.14 . This demonstrated that the significance of SETX was very unlikely a result of technical artifact, such as systematic sequencing error or imputation bias, because the new feature was a combination of hundreds of singletons. It also highlighted that collapsing singletons may increase power when studying association of rare variants using a relatively small sample size. Third, we took a closer examination of allele frequencies of the 380 rare variants located in SETX. Of the rare variants, 92 could be found in the 1000 Genomes Project (2012 February release, http://www.1000genomes. org/). We found their allele frequencies in general population were extremely low (mean frequency $=0.0004$ for 92 rare variants), indicating that these variants were so rare that they may collectively have a composite effect of $\mathrm{OR}=8.8$ while missed in previous studies.

Finally, to further remove possible confounding effect of population stratification, we performed a principal component analysis on 100,000 randomly selected common variants with no missing value and MAF $>0.1$. Logistic regression test was then conducted on the created super feature for SETX, together with the first 10 principal components as covariates. We found that the super feature remained significant, with a $p$ value $=6.7 \times 10^{-5}$, while the 10 principal components were not.

The protein encoded by SETX contains a DNA-RNA helicase domain at its $\mathrm{C}$-terminal, suggesting its involvement in both DNA and RNA processing. Mutations in SETX have been reported to be associated with ataxia-ocular apraxia-2 (AOA2) [13] and an autosomal dominant form of juvenile amyotrophic lateral sclerosis (ALS4) $[10,14]$. However, the function of SETX and its role in hypertension remains unclear and may be worthy of further investigation.

\section{Discussion}

We performed 3 rare-variant association tests for the analysis of a whole genome sequencing data set to identify susceptibility genes in hypertension. We grouped and collapsed rare variants in a gene-based manner for 2 reasons: (a) the deleteriousness of variants could come from protein-coding sequence changes or noncoding intronic regions that contain regulatory elements. (b) Based on the previous simulation study [7], the power of the analysis could be as low as 0.2 (sample size $<200$ ). By collapsing singletons, one may benefit from increasing power. This idea was essentially similar as those burden tests for rare copy number variation in GWAS and de novo mutations in sequencing study. Indeed, we found that the signal was mainly from the intronic regions of SETX in a collective manner of those singletons.

The analysis can be extended and improved in several ways. First, it was shown in qMSAT [7] that incorporation of genotype call qualities directly in association tests can increase power. If raw reads are available, we may call variants and obtain genotype quality information at the same time, using variant calling toolkits $[15,16]$ to further increase our analysis power. Second, we only analyzed rare variants. The association test could be also performed by combining both rare and common variants. Third, we only included 103 unrelated individuals. We may consider adding more samples to increase power. Finally, we focused on only genic regions using conventional gene annotation, which make up little more than $1 \%$ of the genome. The recent annotation made by the ENCODE consortium has included more than 70,000 "promoter" regions and nearly 400,000 "enhancer" regions that regulate expression of distant genes, which account for roughly $80 \%$ of the 
genome [17]. We may utilize this new knowledge in future analysis.

\section{Competing interests}

The authors declare that they have no competing interests.

\section{Authors' contributions}

WW conducted statistical analyses; WW and ZW drafted the manuscript. All authors read and approved the final manuscript.

\section{Acknowledgements}

The authors would like to thank the Genetic Analysis Workshop 18 (GAW18) for preparing and providing the whole genome sequencing data set. We also thank Dr. Pingzhao Hu for the comments and suggestions on the analysis. We thank the 2 reviewers and Dr. Heather Cordell for thei comments and advice, which helped to improve the presentation of the paper.

The GAW18 whole genome sequence data were provided by the T2DGENES Consortium, which is supported by NIH grants U01 DK085524, U01 DK085584, U01 DK085501, U01 DK085526, and U01 DK085545. The other genetic and phenotypic data for GAW18 were provided by the San Antonio Family Heart Study and San Antonio Family Diabetes/Gallbladder Study, which are supported by NIH grants P01 HL045222, R01 DK047482, and R01 DK053889. The Genetic Analysis Workshop is supported by NIH grant R01 GM031575.

This article has been published as part of BMC Proceedings Volume 8 Supplement 1, 2014: Genetic Analysis Workshop 18. The full contents of the supplement are available online at http://www.biomedcentral.com/bmcproc/ supplements/8/S1. Publication charges for this supplement were funded by the Texas Biomedical Research Institute.

Published: 17 June 2014

\section{References}

1. Manolio TA, Collins FS, Cox NJ, Goldstein DB, Hindorff LA, Hunter DJ, McCarthy MI, Ramos EM, Cardon LR, Chakravarti A, et al: Finding the missing heritability of complex diseases. Nature 2009, 461:747-753.

2. Bodmer W, Bonilla C: Common and rare variants in multifactorial susceptibility to common diseases. Nat Genet 2008, 40:695-701.

3. Pritchard JK: Are rare variants responsible for susceptibility to complex diseases? Am J Hum Genet 2001, 69:124-137.

4. 1000 Genomes Project Consortium: A map of human genome variation from population-scale sequencing. Nature 2010, 467:1061-1073.

5. Cooper GM, Shendure J: Needles in stacks of needles: finding diseasecausal variants in a wealth of genomic data. Nat Rev Genet 2011, 12:628-640.

6. Wu MC, Lee S, Cai T, Li Y, Boehnke M, Lin X: Rare-variant association testing for sequencing data with the sequence kernel association test. Am J Hum Genet 2011, 89:82-93.

7. Daye ZJ, Li H, Wei Z: A powerful test for multiple rare variants association studies that incorporates sequencing qualities. Nucleic Acids Res 2012, 40:e60

8. Neale BM, Rivas MA, Voight BF, Altshuler D, Devlin B, Orho-Melander M, Kathiresan S, Purcell SM, Roeder K, Daly MJ: Testing for an unusual distribution of rare variants. PLOS Genet 2011, 7:e1001322.

9. Li B, Leal SM: Methods for detecting associations with rare variants for common diseases: application to analysis of sequence data. Am J Hum Genet 2008, 83:311-321.

10. Pruitt KD, Tatusova T, Maglott DR: NCBI Reference Sequence (RefSeq): a curated non-redundant sequence database of genomes, transcripts and proteins. Nucleic Acids Res 2005, 33:D501-D504.

11. Pan W: Asymptotic tests of association with multiple SNPs in linkage disequilibrium. Genet Epidemiol 2009, 33:497-507.

12. Wang K, Li M, Hakonarson H: ANNOVAR: functional annotation of genetic variants from high-throughput sequencing data. Nucleic Acids Res 2010, 38:e164.

13. Arning L, Schols L, Cin H, Souquet M, Epplen JT, Timmann D: Identification and characterisation of a large senataxin (SETX) gene duplication in ataxia with ocular apraxia type 2 (AOA2). Neurogenetics 2008, 9:295-299.
14. Chen YZ, Bennett CL, Huynh HM, Blair IP, Puls I, Irobi J, Dierick I, Abel A, Kennerson ML, Rabin BA, et al: DNA/RNA helicase gene mutations in a form of juvenile amyotrophic lateral sclerosis (ALS4). Am J Hum Genet 2004, 74:1128-1135.

15. McKenna A, Hanna M, Banks E, Sivachenko A, Cibulskis K, Kernytsky A Garimella K, Altshuler D, Gabriel S, Daly M, et al: The Genome Analysis Toolkit: a MapReduce framework for analyzing next-generation DNA sequencing data. Genome Res 2010, 20:1297-1303.

16. Wei Z, Wang W, Hu P, Lyon GJ, Hakonarson H: SNVer: a statistical tool for variant calling in analysis of pooled or individual next-generation sequencing data. Nucleic Acids Res 2011, 39:e132.

17. Dunham I, Kundaje A, Aldred SF, Collins PJ, Davis CA, Doyle F, Epstein CB, Frietze S, Harrow J, Kaul R, et al: An integrated encyclopedia of DNA elements in the human genome. Nature 2012, 489:57-74.

doi:10.1186/1753-6561-8-S1-S50

Cite this article as: Wang and Wei: Collapsing singletons may boost signal for associating rare variants in sequencing study. BMC Proceedings 2014 8(Suppl 1):S50.

\section{Submit your next manuscript to BioMed Central and take full advantage of:}

- Convenient online submission

- Thorough peer review

- No space constraints or color figure charges

- Immediate publication on acceptance

- Inclusion in PubMed, CAS, Scopus and Google Scholar

- Research which is freely available for redistribution
C Biomed Central 